\title{
Formas organizacionales feministas en la implementación de políticas públicas
}

\author{
Silvia Cristina Caicedo-Muñoz' (iD 0000-0003-1 108-5305 \\ 'Universidad de San Buenaventura, Cali, Colombia.sccaiced@usbcali.edu.co
}

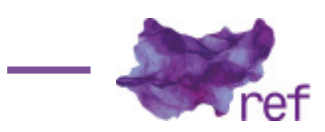

\begin{abstract}
Resumen: Este artículo contribuye al estudio de la implementación de políticas públicas a partir de los estudios organizacionales asociados a los estudios de género, al analizar cómo las organizaciones no gubernamentales de desarrollo formadas por mujeres se organizan para implementar políticas públicas a partir de tensiones, prácticas deliberativas y conflicto.

El estudio de caso múltiple basado en entrevistas semiestructuradas permite afirmar que las organizaciones de mujeres establecen "formas organizacionales feministas", por tanto, no son organizacionalmente disonantes, no adaptan el tipo burocrático ideal, no son sexualizadas, ni reproducen diferencias de género.
\end{abstract}

Palavras clave: políticas públicas; implementación; organización; género; empoderamiento.

Formas organizacionais feministas na implementação de políticas públicas

Resumo: Este artigo contribui para o estudo da implementação de políticas públicas baseadas nos estudos organizacionais associados aos estudos de gênero, analisando como as organizações nãogovernamentais de desenvolvimento formadas por mulheres se organizam para implementar políticas públicas baseadas em tensões, práticas deliberativas e conflitos.

O estudo de casos múltiplos baseado em entrevistas semiestruturadas permite afirmar que as organizações de mulheres estabelecem "formas organizacionais feministas", portanto, não são dissonantes organizacionalmente, não adaptam o tipo burocrático ideal, não são sexualizadas, nem reproduzem diferenças de gênero.

Palavras-chave: políticas públicas; implementação; organização; gênero; empoderamento.

Feminist organizational forms in the implementation of public policies

Abstract: This article contributes to the study of the implementation of public policies based on the organizational studies associated with gender studies, by analysing how non-governmental development organizations formed by women organize themselves to implement public policies based on tensions, deliberative practices and conflict.

The multiple case study based on semi-structured interviews allows to affirm that women's organizations establish "feminist organizational forms", therefore, they are not organizationally dissonant, they do not adapt the ideal bureaucratic type, they are not sexualized, not do they reproduce gender differences. Keywords: Public policy; Implementation; Organization; Gender studies; Empowerment.

\section{Introducción}

Las mujeres participan políticamente desde los 70 ìs en el desarrollo social, a través de las organizaciones de movimientos sociales, y no gubernamentales de desarrollo social ONGD ìs. En América Latina, Sonia Alvarez y Maria Ceci Misoczky mencionan que algunas ONGD ìs han generado el fenómeno de ongenización, en el cual se presentan tensiones y contradicciones (Jean BARTUNEK; Kate WALSH; Catherine LACEY, 2000) con respecto a las políticas públicas, por la adaptación de procesos y estructuras organizativas similares a las de las públicas o del Estado (Karen ASHCRAFT, 2001, 2009; Maria Ceci MISOCZKY; Rafael Kruter FLORES; Sueli Maria Goulart SILVA, 2008; Stephanie 
RIGER, 1994); o porque establecen una relación entre género y organización dirigida a agregar mujeres en las organizaciones (Matts ALVESSON; David STANLEY, 2006; Dennis MUMBY; Linda PUTNAM, 1992; ASHCRAFT; MUMBY, 2004).

El desarrollo social, operativizado en políticas públicas, implica reflexionar las formas de organización en grupos que históricamente generan transformaciones sociales en su bienestar, y que han permanecido marginales en la literatura, como son las ONGD de mujeres. Al respecto, los estudios organizacionales de género señalan, entre muchas vertientes, que las mujeres se organizan igualitariamente y generan participación e intimidad con estructuras jerárquicas formales (RIGER, 1994). No obstante, este artículo presenta un estudio de caso múltiple (Robert YIN, 2003) que genera reflexividad (Lisa COSGROVE; Maureen MCHUGH, 2000) de las mujeres en contextos cotidianos en dos ONGD's locales en Cali, integradas y lideradas por ellas, con más de 5 años de existencia, y coexistentes a organizaciones comunitarias de base en su gestión. Pretende responder la pregunta por ¿Cómo se organizan las mujeres que integran ONG para implementar políticas públicas?, indagar las formas organizacionales y las creencias ideológicas feministas que generan empoderamiento e inciden en la implementación de políicas, las características de la intervención social, y cómo son los procesos y los resultados de empoderamiento psicológico, comunitario y organizacional, en tres niveles de análisis estructural, operativo y subjetivo.

El estudio de caso se construyó a partir de guías, manuales, protocolos, informes, librosguía; entrevistas semiestructuradas a 22 mujeres entre directivas, y consejeras de familia (equipo en terreno y coordinadoras de programa) de la ONGD 1 y a 3 mujeres de la ONGD 2, así como, cuatro observaciones no participantes y dos grupos de discusión, los cuales permitieron registrar cotidianamente la acción y emociones de las mujeres y validar los hallazgos con ellas, respectivamente. La unidad de análisis es la organización, pues hay diferencias estructurales y culturales con relación a la opresión política y personal a las que se exponen, y existen intersecciones mitificadas de género, sexualidad, raza, etnicidad y clase relacionadas con su rol en la política pública.

Según los resultados, las ONGD's de mujeres que coexisten con un movimiento social en su gerencia, no son disonancias organizadas (ASHCRAFT, 2001), ni son genderizadas (Joan ACKER, 1990, 2006; Danna BRITTON, 2000). Son formas femeninas contextualizadas, histórica, social, y políticamente a los procesos de empoderamiento organizacional y psicológico que cuestionan empíricamente desde una posición feminista (RIGER, 1994) al management del desarrollo y al empoderamiento como estrategia de éxito de las intervenciones sociales.

\section{Las ONGD's de mujeres, el empoderamiento y las políticas públicas}

Las mujeres son consideradas los mayores agentes del desarrollo económico (Shara NEIDELL, 1998), en el "gender and development" (Michael BAMBERGER; Donna PODEMS, 2002), sus formas de organización permiten analizar la prestación de servicios sociales en el ciclo de vida de las políticas públicas, especialmente con las ONGD ìs, debido a su eficacia, las intervenciones sostenibles, el voluntariado y la credibilidad (Alan FOWLER, 2000).

En Latinoamérica, algunas mujeres de movimientos sociales conformaron organizaciones feministas (NEIDELL, 1998), afrontando tensiones y contradicciones comunicativas (BARTUNEK; WALSH; LACEY, 2000) al adoptar procesos y estructuras similares a las organizaciones públicas o del Estado. Otras se fortalecieron con el apoyo de organismos multilaterales, a partir de la promoción del empoderamiento como estrategia exitosa (Michael EDWARDS; David HULME, 1996) en desarrollo social (NEIDELL, 1998). Aunque, algunas activistas y los nuevos movimientos sociales hayan cuestionado el empoderamiento, dada la marginalización y su impacto en las subjetividades, sugieren reempoderarlas para liberar su agencia (Jenny PEARCE, 2013). Este panorama implica explorar la relación entre los estilos de liderazgo de la mujer y el empoderamiento de los miembros en organizaciones con principios feministas e ideología igualitaria (RIGER, 1994), pues enfrentan el dilema de cómo empoderar.

Las mujeres como fuerza políica tienen variedad ideológica y organizacional, y son las circunstancias de su constitución como organización lo que les hace singulares (Joanne MARTIN, 1990) y les hace cambiar. Los estudios de las organizaciones feministas se enfocan en la estructura interna, pero Patricia Yancey Martin (1990) considera que eso no determina su esencia feminista pues muchas fueron creadas por movimientos sociales de liberación de la mujer; la organización feminista tiene metas, estrategias e ideologías feministas y valores en función de transformar la autoestima, la conciencia política, las habilidades y el conocimiento; proveer educación política y consejería; así como, generar cambio en el trato, oportunidades y condiciones de vida de las mujeres. Luego, son comparables en ideología, valores, metas, productos, circunstancia de constitución, estructura, prácticas, miembros, alcance, escala, relaciones externas, y estrategias.

En las organizaciones de mujeres por adaptación o innovación organizativa, las formas burocráticas pueden existir o no, coexistir con las nuevas, o estar asociadas a procesos de burocratización estatal. Esto puede configurar la ongenización (ALVAREZ, 1999) de las 
organizaciones que históricamente cuentan con la existencia de movimientos sociales, pues adoptan formas burocráticas o coexisten con las creadas (ASHCRAFT, 2001, 2009; RIGER, 1994) y cuestionan la intersección de los estudios del género y de las organizaciones, con base en la racionalidad burocrática que masculiniza lo público desde la feminización de lo privado o genera intervenciones sostenibles producto de la feminización de la organización. En esa vía, algunas investigaciones en Colombia concluyen que el cómo se generó la estructura organizacional, la acción, esquemas interpretativos y normas consensuadas de sus integrantes dependen del empoderamiento e inciden en el cambio social en los procesos comunitarios de mujeres que implementan políticas exitosamente.

\section{Los estudios organizacionales de género en la implementación de políticas públicas}

El carácter simbólico de las políticas públicas implica el estudio crítico de quienes la ejecutan, usar la interpretación del discurso y el análisis narrativo (John FORESTER, 2008); y reconocer los modelos de implementación de políticas (Laurence O ìTOOLE; Robert MONTJOY, 1984; Peter DELEON; Linda DELEON, 2002). El modelo top-down, centrado en comprender la causa-efecto de las decisiones desde la visión racional, supone que la implementación es la acción de dos actores opuestos, en procesos burocráticos y políticos con metas pre estructuradas desconociendo actores y la ajustabilidad de las políticas. Por su parte, el modelo bottom-up afirma que la implementación depende de quienes la ejecutan -burócratas en terreno - o los beneficiarios, según el tiempo, las políticas, las unidades del gobierno, las relaciones formales o no, y los subsistemas de políticas. Mientras que el enfoque mixed-scanning o de exploración combinada con tendencias democráticas ofrece una visión científica según las características de la implementación a partir de hipótesis variables, predicción del comportamiento desde la teoría de juegos y la agencia en estudios de caso comparado; así como reconoce las tensiones entre los procesos y las estructuras organizacionales en la acción.

Las investigaciones sobre la implementación de políticas públicas han fragmentado los análisis empíricos (Michael HILL; Peter HUPE, 2002) y aluden al diseño (top-down, bottom- up, o interorganizacional), pero no distinguen las dimensiones organizacional, administrativa, financiera y sociocultural. Lo organizacional posibilita explorar, describir, analizar y comprender la implementación a partir de la agencia y la colaboración intraorganizacional (O iTOOLE; MONTJOY, 1984) con visión crítica de los significados y significaciones históricas y subjetivas (FORESTER, 2008). Los enfoques bottom-up y top- down no incluyen referencias sobre la definición de las políticas locales o del sistema político-administrativo, por ende, en el ejercicio normativo debe diferenciarse lo teórico de lo metodológico para identificar las situaciones en las cuales la colaboración es central en la actividad política; así como el lugar en la implementación de los recursos y los resultados, de la relación diseñador-implementador, de los procesos en terreno, y de las relaciones interorganizacionales (HILL; HUPE, 2002) y lo organizacional (Cyril BLACK; John BURKE, 1983) en las intervenciones sociales.

Los estudios de la implementación de políticas públicas y los estudios de género se han abordado en Latinoamérica desde el análisis de transferibilidad de políticas públicas, se han vinculado a la reforma de la administración pública y la desregulación del Estado, la evaluación para comprender los procesos de implementación y de diseño de políticas públicas afirmativas (Carlos Rodrigues da SILVA, 2010), el uso de modelos estructuralistas europeos y anglosajones en las organizaciones de la administración pública (Lucas Canestri de OLIVEIRA; José Roberto PEREIRA; Patricia Aparecida FERREIRA, 2013), el estudio crítico de la gestión de acciones dirigidas al género (Elisângela Abreu NATIVIDAD; PEREIRA; Vânia Aparecida Rezende de OLIVEIRA, 2013).

Sin embargo, reflexionar sobre los estudios de la implementación, de las organizaciones y del género es reconocer que la teoría de las organizaciones constituye una posibilidad intelectual en el estudio de las políticas pues se apoya en los conceptos de poder, estrategia y sistema organizado, donde el todo es más que la suma de los miembros (Juliana MEJÍA, 2012), por ejemplo, en los estudios del diseño de políticas no son relevantes los movimientos sociales (Martha FARAH, 2004), y en otros casos, las políticas públicas para mujer se asocian al empoderamiento producto del cambio institucional en relaciones de género, aún no dadas (Cecília SARDENBERG, 2008).

En la psicología comunitaria la participación de la comunidad en las políticas se asocia al diseño e implementación (Maritza MONTERO, 2009). Constituye un campo a explorar, en el cual la intervención social es el vínculo principal en pro del cambio, el empoderamiento es un proceso de movilización social intencional comunitario para desarrollar capacidades basadas en el respeto y la reflexión (David PERKINS; Marc ZIMMERMAN, 1995), y la participación comunitaria en la solución de sus problemas sociales facilita el seguimiento a las acciones de las ONG, y el Estado mide el impacto e identifica las coaliciones público-privadas con la comunidad (Stephen FAWCETT; Adrienne PAINE-ANDREWS, 1999)

En el estudio de la implementación de las políticas públicas, las organizaciones y sus roles se 
comprenden desde: a) la agencia y la colaboración intraorganizacional (O iTOOLE; MONTJOY, 1984), b) el impacto de los actores en los procesos de formación de políticas públicas, la participación ciudadana, democracia local y en el diseño de políticas (HILL; HUPE, 2002). Sin embargo, la aproximación al fenómeno requiere conceptos de los estudios organizacionales de género.

Las mujeres tienden a organizarse de manera igualitaria, con una estructura jerárquica formal que posibilita participación e intimidad, prefieren manejos consensuados y equipos que tomen decisiones (RIGER, 1994), especialmente aquellas con principios feministas, pues el empoderamiento es equitativo. La burocracia como una expresión estructural de la dominación masculina genera colectivismos híbridos o disonancias organizadas entre las feministas, pues funcionan con estructuras colectivas o poseen problemas, producto de las tensiones entre valores organizacionales compartidos por los miembros y las demandas cotidianas de una organización en crecimiento. Las estructuras burocráticas híbridas entre feministas facilitan la movilización de modos de poder y de igualitarismo, logrando legitimidad. Es la unión estratégica de formas presuntamente hostiles, para capturar la ironía de lo alterno (ASHCRAFT, 2001)

Mientras que para Riger (1994), la burocracia es una forma de organizarse, y no por ello las organizaciones que la adoptan son antifeministas; por el contrario, responden a su crecimiento organizacional, que da cuenta del micro orden social y de las estrategias para desarrollar habilidades y cooperar. La necesidad de mostrar legitimidad puede poner a una organización feminista con tendencia a ser más burocrática y a tener cooperación formal, en camino de ser aceptada como institución, de afrontar dilemas por conflictos entre necesidades individuales y organizacionales, por decidir entre generar participación o productividad o por el ingreso de integrantes con valores diversos. La burocracia femenina en organizaciones feministas muestra la influencia de otras organizaciones y las tensiones internas por no ceder ideológicamente ni incorporar las reconfiguraciones de las necesidades individuales en las prácticas formales, contrario a lo sucedido con la organización burocrática, en la cual las necesidades emocionales y los sistemas sociales informales resuelven la frustración por vía de la represión individual. De hecho, algunas feministas consideran que las organizaciones burocráticas inevitablemente oprimen al trabajador, mientras que la burocracia se constituye como estructura beneficiosa para las organizaciones feministas al facilitar el logro de metas, generación de justicia y accountability, siendo éste último el que posibilita abuso de poder a través de la burocracia, luego, las organizaciones feministas pueden adherir a visiones alternativas basadas en formas burocráticas (RIGER, 1994), y solo la burocracia emancipadora maximiza el poder, aunque con costo práctico y ético (ASHCRAFT, 2001).

Las posiciones de Ashcraft y Riger exponen el dilema que enfrentan las organizaciones feministas, dado que la/s racionalidad/es y el sujeto omnisciente administrativo se sobreponen a los valores e identidad de las organizaciones feministas que implementan políticas; o las formas racionales se evidencian en el empoderamiento comunitario debido a que la participación y el colectivismo son diseñados para generar capacidades de empoderamiento a través de la equidad y el control; o se establecen impersonalidades burocráticas orientadas a la mujer como sujeta política en relaciones igualitarias; se adoptan diversas formas comunicativas marcadas por las emociones, ${ }^{\prime}$ como lo señalan Mamby y Putnam (1992, p. 473); o se generan disonancias organizacionales (ASHCRAFT, 2001) producto de la alineación de las creencias ontológicas de cada mujer con la vida organizacional (Cliff OSWICK; PUTNAM; Tom KEENOY, 2004).

Por lo anterior, es necesario comprender la naturaleza dinámica de la democracia deliberativa ${ }^{2}$ en las prácticas colectivas barriales, ya que la gobernanza se genera por la relación entre conflicto constructivo e integración y la ley de la situación y el comportamiento circular (María ELIAS, 2010). El conflicto es natural, puede ser constructivo en los humanos y es inevitable en los procesos comunitarios (Mary Parker FOLLETT, 1919, 1925), ya que es una actividad unificada continúa, recreable por ideas y por el proceso de síntesis de la interacción humana, que genera empoderamiento. Luego, las prácticas deliberativas constituyen conocimiento colectivo por debate político entre los integrantes producto de la concientización, y aportan a resolver los problemas eficiente y pragmáticamente, pues cada comunidad tiene procesos colectivos y formas de "governance" (ELIAS, 2010) únicos.

El empoderamiento (PERKINS; ZIMMERMAN, 1995) se entiende en tres niveles: a) Psicológico o individual, que alude a la percepción de control personal, actitud proactiva hacia la vida y comprensión crítica del ambiente sociopolítico, aprendiendo habilidades para tomar decisiones, manejar recursos y trabajar con otros; b) Organizacional, son procesos y estructuras que favorecen

\footnotetext{
' Las emociones están contenidas en símbolos (mitos e historias) que trasmiten los valores organizacionales y las ideologías; y facilitan la toma de decisión y la identificación organizacional a través de la alineación de las emociones de los integrantes con la racionalidad de la organización.

${ }^{2}$ La democracia deliberativa incluye la ciudadanía democrática y las prácticas deliberativas. La primera es la constitución colectiva de conocimiento por deliberación política grupal; la segunda son las acciones de los integrantes de los barrios que logran cambios en la calidad de los servicios, producto de la concientización y planeación de la solución a los problemas eficiente, pragmática y única, dado que cada comunidad tiene procesos colectivos únicos con su propia forma de gobierno.
} 
las estrategias de los sujetos y el apoyo mutuo en el manejo de recursos y de redes de coalición organizacional, por la participación en las decisiones, responsabilidades y liderazgo; y c) Comunitario, es la acción colectiva de los individuos en organizaciones para mejorar la calidad de vida y las relaciones con la comunidad. Esta desarrolla habilidades para acceder a recursos propios, con actitud de apertura a estructuras mediadoras y tolerantes a la diversidad, y produce coaliciones con liderazgo plural y participación comunitaria.

La implementación en lo político y lo técnico plantea retos y dificultades entre las organizaciones y sujetos, y presenta problemas de sostenibilidad, pues muchas veces el diseñador es distinto del implementador; las concepciones ideales de sociedades racionalizadas inciden en la toma de decisión; y el diseño de la intervención no coincide con los problemas del contexto. Por lo tanto, cuando la implementación es por apropiación y acción directa de las comunidades, se deben trascender los modelos y enfoques, en particular si son problemas sociales contemporáneos (salud mental, género, crimen y seguridad), con procesos transformadores basados en la acción por acuerdos cognitivos entre actores, en torno a los problemas comunitarios e impacta la realidad desde/en/para la comunidad empoderada (MONTERO, 2009).

\section{Análisis de datos}

Las ONGD's seleccionadas son representativas del tercer sector en Colombia, pues implementan políticas públicas con acciones desde una comunidad de base, tienen financiación internacional y pública, su fin es el empoderamiento, y la administración de los programas de la ONGD 1 la realizan mujeres de la comunidad y en la ONGD 2 lo hacen el equipo base y el de activistas feministas. Fueron creadas al final de los años 80 y reconocidas jurídicamente en los años 90 , período de influencia del modelo neoliberal y de inclusión del concepto de empoderamiento. Las prácticas administrativas y financieras son eficaces, abordan problemas sociales no suplidos por el Estado, aportan a la definición de la agenda pública (EDWARDS; HULME, 1996) y a la ejecución, monitoreo y evaluación de políticas; tienen discursos alternativos de desarrollo y democratización de los servicios sociales; no alinean sus acciones a los requerimientos de los gobiernos locales; participan en redes formales e informales y en coaliciones comunitarias. Las ONGD's se analizan longitudinalmente en tres niveles de análisis: estructural, operativo y subjetivo entre los períodos 1989 a 2017 y 1999 a 2017.

Nivel 1. Estructural. Se analizan y comparan los conflictos y adaptación en los momentos de gestión clave en la ejecución de proyectos y en procesos comunitarios, según Parker, con la estrategia narrativa (Ann LANGLEY, 1999). Las ONGD's amplían sus acciones y el número de integrantes; en la ONGD 1, de 20 a 60 Consejeras de Familia, profesionales y personal administrativo, mientras que a la ONGD 2 se suman activistas feministas a las acciones políticas en espacios públicos.

La ONGD 2 no posee un estilo burocrático con formas disonantes organizacionales (ASHCRAFT, 2001), pues se organizan horizontalmente.

(...) yo creo que el feminismo, propone una mirada del mundo especial, en donde las mujeres estamos inconformes con la forma en que está el mundo, donde las mujeres tenemos una voz invisibilizada, donde los derechos de las mujeres, pues están en primer orden, en donde la agenda de las mujeres, que son nuestros deseos, nuestras luchas, no están representadas en público, y en todo el quehacer del Estado. (...) todo lo de mi cuerpo es mío, como el placer de la mujer hay que agenciarlo y hacer visible, donde la mujer queremos estar en la esfera pública, donde las mujeres necesitamos ganar empoderamiento. Eso es lo que agenciamos, tener relaciones horizontales, fue como una de las primeras cosas, o sea intentar hacer intuitivamente, porque ya desde la realidad una organización que fuera horizontal, en donde no hubiera una directora, sino que tuviéramos todo un que hacer desde las potencialidades y no desde las jerarquías, eso fue intuitivo porque ninguna sabía. (Directiva 2, 2017)

La conscientización social y política de las mujeres encuentra sentido organizacional y comunitario:

el feminismo da una manera de acercarse al mundo desde otros ojos, (...) nos parece natural porque el mundo está en masculino, nos habituamos a eso (...), el lenguaje que desde el feminismo se agencia es porque es una manera de cambiar la realidad. Entonces digamos que el feminismo le da también otras maneras de ser más críticos y de pensar que las cosas como están no están bien y que uno las quisiera cambiar y de alguna manera incidir en ese cambio pues se vaya dando; al menos darnos cuenta ya es un gran paso y pensar en que lo tenemos que transformar y que queremos que no siga siendo más así, también es otro paso, pero al menos darnos cuenta y pensar que eso no es natural, que eso no está dado. (Directiva, 2017)

El enfoque de las intervenciones sociales está marcado por la incorporación de las estrategias comunicativas en los barrios, grupos, comunidades para generar empoderamiento "Entonces empezamos a trabajar desde la comunicación, a partir de estrategias de información, educación; era para empoderar un poco a las comunidades" (Directiva, 2017). 
Ambas ONGD's basan sus relaciones en conflictos de carácter restaurativo entre ellas o con los donantes, ya que la acción conjunta se desarrolla en contextos particulares, como negarse a continuar la ejecución de proyectos relacionados con políticas públicas criticadas por su enfoque de intervención. En la ONGD 2 se potencializa la sincronización de las emociones generadas en las integrantes el asumir un proyecto; es decir, supone la existencia de formas de cooperación sensibles a las acciones de otros. Tal como se muestra en el Cuadro 1.

\section{Cuadro 1}

\begin{tabular}{|c|c|c|c|}
\hline \multicolumn{2}{|c|}{ CATEGORIA } & ONGD 1 & ONGD2 \\
\hline \multicolumn{2}{|c|}{ Conflieto (Follett, 192.5) } & Conflicto asumido como natural & Paede ser construactivo \\
\hline \multicolumn{2}{|c|}{$\begin{array}{l}\text { Procesos comunitarios (Follett, } \\
1918,1919 \text { ) }\end{array}$} & $\begin{array}{l}\text { Trabajo en red: CF por nodas. } \\
\text { Lis CF son mujeres de la comunidad intervenida } \\
\text { Actividades entorno a la común-unidad }\end{array}$ & $\begin{array}{l}\text { Artivimos: No son indegrantes } \\
\text { permanentes de la ONGD }\end{array}$ \\
\hline \multirow{5}{*}{$\begin{array}{l}\text { Fstile de } \\
\text { burocracia } \\
\text { formas } \\
\text { disenastes } \\
\text { organizacionales } \\
\text { (Asbcraft, 2001), }\end{array}$} & $\begin{array}{l}\text { Metas } \\
\text { primarias/ } \\
\text { Racionalidad }\end{array}$ & $\begin{array}{l}\text { Organización como medio y fin: eficiente, productiva, } \\
\text { y el empoderamiemto consciente del género. }\end{array}$ & $\begin{array}{l}\text { Organización como fin: el } \\
\text { empoderamienoo de la majer. }\end{array}$ \\
\hline & $\begin{array}{l}\text { Estructara de } \\
\text { poder }\end{array}$ & \multicolumn{2}{|c|}{$\begin{array}{l}\text {-Igaalitaria o heterirquica. } \\
\text {-Autoridad descentralizada y fundamentada en la toma de decisiones consensuadas } \\
\text { colectivamente }\end{array}$} \\
\hline & Roles & \multicolumn{2}{|c|}{$\begin{array}{l}\text { - Pocas roles } \\
\text { - Roles informales } \\
\text { - Particularismo subjetivo (Aplicación y negociación situacional) } \\
\text { - Control vía preferiblemente por creencias compartidas }\end{array}$} \\
\hline & $\begin{array}{l}\text { División del } \\
\text { trabajo }\end{array}$ & $\begin{array}{l}\text { - Informal } \\
\text {-No especializada } \\
\text {-Rotación de tareas. }\end{array}$ & $\begin{array}{l}\text {-Fonmal e informal } \\
\text {-Tareas definidas/flexibilidad } \\
\text { dialéctica. }\end{array}$ \\
\hline & $\begin{array}{l}\text { Cualificacione } \\
\$ \text { para la }\end{array}$ & $\begin{array}{l}\text {-Avance personal regulado por el Bien común. } \\
\text {-Metas de los avances individuales son reemplazados } \\
\text { por la construcción de comunidad }\end{array}$ & $\begin{array}{l}\text {-Avance personal regulado por el } \\
\text { bien común. }\end{array}$ \\
\hline
\end{tabular}

Fuente: Elaboración propia

\#PraCegoVer Cuadro 1 presenta tres columnas, Categoría, ONGD1 y ONGD2, con las conductas de cada una de las ONGDs, en nivel estructural, en cuanto a 3 categorías: Conflicto; Procesos comunitarios; Estilo de burocracia - formas disonantes organizacionales.

Nivel 2. Operativo. Se reconstruyen los períodos y cambios de la intervención social y la estructura organizacional durante 5 años, a partir de las entrevistas semiestructuradas y la revisión documental, con una estrategia de análisis narrativa. La información recopilada en las visitas de campo se compara para realimentar puntos comunes y válidos (YIN, 2003) del relato en las categorías de identidad y conocimiento local; valores; ciudadanía democrática y prácticas deliberativas; dilemas de los integrantes en la organización y tácticas para resolverlos, emociones y relaciones.

La ONGD 1, de origen franciscano, establece su intervención bajo la estrategia "Comunidades religiosas insertas en medios populares" dirigida a poblaciones étnicamente plurales y la teología de la liberación que forma comunidades eclesiásticas de base (...) en pequeños grupos autónomos y libres, capaces de gestionar de acuerdo al evangelio, trabajar desde ellos y no para ellos, es un diálogo de saberes de iguales en el que la comunidad académica no es más que ellos" (Directiva, 2009); con el propósito de recuperar la palabra, especialmente las mujeres: "un esfuerzo muy grande para que las gestores del proceso fueran las mujeres (...) ninguna de ellas está por un salario, hay otra motivación, es de guía espiritual, eso hace que sea distinto" (Directiva 2, 2009).

En los años 80, su labor social estuvo bajo el principio de la "enajenación de las personas" y los fundamentos filosóficos de Amarthya Sen, que subrayan la relevancia de poder elegir, como se menciona "la gente piensa en términos de asistencialismo y el Distrito de Aguablanca nació a la espalda de Cali, es una zona de la ciudad que está estigmatizada. Nos tocó con ayuda internacional suplir necesidades básicas" (Directiva, 2009). Por ello, los problemas públicos y sociales son definidos desde la realidad social de la comunidad, a partir del concepto de necesidad:

todos los programas surgieron de aquí por necesidad, si!!, esto fue una invasión que vio la Hermana, reunió a todas las mujeres y les ofreció alternativas, empezamos nosotras las mujeres jóvenes y los muchachos en grupitos para apoyarnos, bueno de ahí llegaron las 7 mujeres que empezamos y nos reuníamos ahí. Ella siempre ha hablado de que las mujeres tenían derechos, derechos a trabajar. Nosotras las mujeres teníamos derecho a educarnos, a una vida digna. (Consejera de Familia 1, 2009) 
Por su parte, en la ONGD 2 se consolida la perspectiva de la mujer en el discurso de los derechos humanos, la comunicación, la salud, derechos sexuales y reproductivos, la participación política, los modelos pedagógicos y las agendas informativas, a través de estrategias de participación como los Artivismos "Como al activismo hay que ponerle arte, entonces artivismos. Entonces hay que ponerle poesía al activismo (...) es hacer otras acciones, otras puestas en escenas otras temáticas que apunten a lo artístico a la vida" (Directiva, 2017). Las prácticas de relación entre las integrantes están mediadas por el activismo, y convergen prácticas deliberativas sobre las acciones públicas a desarrollar.

no ofrecemos servicios (...) pertenecemos a la red Nacional de mujeres, digamos que más que un voluntariado, lo que tenemos son las activistas, Entonces ahí tenemos un grupo de mujeres que son claves, porque entonces usted tiene que llamar Siempre a estas personas porque son las que vienen a la reunión porque son las que hacen parte de la red de mujeres. Mavi es el centro de toda esa movilización de mujeres de toda la ciudad como núcleo. Entonces no son, digamos en categoría debe de voluntarios si no, de activista entonces también cambia. (Directiva, 2017)

El feminismo constituye el principal valor de la acción de las integrantes de ambas ONGD's. Son socialmente innovadoras (FOWLER, 2000), pues sus intervenciones sociales se sostienen en una estrategia de empoderamiento comunidad-organización, aumentan la autonomía y responsabilidad de las beneficiarias (EDWARDS; HULME, 1996) y mejoran el bienestar (Ver Cuadro 2).

\section{Cuadro 2}

\begin{tabular}{|c|c|c|}
\hline CATEGORIAA & ONGD 1 & ONGD 2 \\
\hline Valores (Asheraft, 2001) & $\begin{array}{l}\text { Solidaridad } \\
\text { Alteridad } \\
\text { Conscientización } \\
\text { Empoderamiento } \\
\text { Feminismo }\end{array}$ & $\begin{array}{l}\text { Solidaridad } \\
\text { Empoderamiento } \\
\text { Comparten el conocimiento } \\
\text { (libre derechos de autor) } \\
\text { Feminismo }\end{array}$ \\
\hline $\begin{array}{l}\text { Identidad y } \\
\text { conocimiento local } \\
\text { (Asheraft, 2001; Riger, } \\
\text { 1994) }\end{array}$ & $\begin{array}{l}\text { La OGND desarrolló la } \\
\text { pedagogia de la liberación de la } \\
\text { opresión de género. }\end{array}$ & $\begin{array}{l}\text { Felicidad } \\
\text { Tamboreadas (Acciones) en } \\
\text { zonas de Cali }\end{array}$ \\
\hline $\begin{array}{l}\text { Ciudadanía democrática } \\
\text { (Follett, 1918) }\end{array}$ & $\begin{array}{l}\text { Integrada por Consejeras de } \\
\text { Familia de la Asociación } \\
\text { Semilla de Mostaza, con su } \\
\text { sistema de gobierno y normas. }\end{array}$ & $\begin{array}{l}\text { Colectivo de mujeres } \\
\text { Artivarte; usan arte, no } \\
\text { activismos }\end{array}$ \\
\hline $\begin{array}{l}\text { Prácticas deliberativas } \\
\text { (Follett, 1929) }\end{array}$ & $\begin{array}{l}\text { Toma de decisiones horizontal } \\
\text { entre la Asociación semilla de } \\
\text { mostaza y la ONGD. } \\
\text { Bienestar comunitario según la } \\
\text { visión de las integrantes de la } \\
\text { ONGD. }\end{array}$ & $\begin{array}{l}\text { Toma de decisiones } \\
\text { horizontal } \\
\text { Colectivo de mujeres }\end{array}$ \\
\hline $\begin{array}{l}\text { Dilemas de los } \\
\text { integrantes en la } \\
\text { organización y tácticas } \\
\text { para resolverlos, } \\
\text { emociones y relaciones. } \\
\text { (Mumby y Putnam, } \\
\text { 1992) }\end{array}$ & $\begin{array}{l}\text { Mito: El árbol de guanábana y } \\
\text { las } 7 \text { mujeres } \\
\text { Historias: Consejera de Familia } \\
\text { que le refutó al Presidente de la } \\
\text { República, sobre economia } \\
\text { social. }\end{array}$ & $\begin{array}{l}\text { Mito: Proyecto de } \\
\text { comunicación internacional } \\
\text { que generó tristeza y estrés } \\
\text { en las integrantes; es } \\
\text { referente para sus decisiones. } \\
\text { Historias: Participación en la } \\
\text { formulación de la Politica } \\
\text { Pública de mujeres de Cali. }\end{array}$ \\
\hline
\end{tabular}

Fuente: Elaboración propia

\#PraCegoVer Cuadro 2 presenta tres columnas, Categoría, ONGD1 y ONGD2, con las conductas de cada una de las ONGDs, en nivel operativo. Son cinco las categorías: Valores; Identidad y conocimiento local; Ciudadanía democrática; Prácticas deliberativas; Dilemas de los integrantes en la organización y tácticas para resolverlos, emociones y relaciones. 
Nivel 3. Subjetivo. Se identifican y caracterizan los momentos y eventos claves de las ONGD ìs locales en 5 años con respecto al empoderamiento psicológico, organizacional y comunitario a través del proceso biográfico-organizacional de las mujeres (Jerome BRUNNER, 1988).

Desde la psicología comunitaria, las ONGD ìs tienen un enfoque basado en la coalición de acción comunitaria (FAWCETT; PAINE-ANDREWS, 1999) debido a las formas de organización de las mujeres, el interés por temas locales, el abordaje de las necesidades de la comunidad usando sus recursos y el establecimiento de alianzas sobre la colaboración y acciones en varios temas. Además, reconfiguran las metas del desarrollo comunitario al captar el significado de los problemas en el contexto, promover el empoderamiento y generar conductas proactivas hacia la política y el cambio social (PERKINS; ZIMMERMAN, 1995).

En perspectiva biográfica organizacional de las mujeres, ambas ONGD's tienen sus propios periodos de empoderamiento, singulares a su forma de organizarse y de actuar. En la ONGD 1 se caracterizaron 3 períodos de empoderamiento orientado por la teología de la liberación del oprimido de género:

Período 1. ONGD 1. Consolidación de las formas organizacionales. Del año 1987 a 1990. La constitución de la ONGD se debe a los discursos de una mujer perteneciente a una organización religiosa sobre el rol de la mujer en la vida cotidiana de las familias, y al cuestionar esquemas de interpretación de la realidad de siete mujeres que se reúnen luego de sus quehaceres a conversar. Se generan procesos de introspección, a partir de artefactos culturales como el árbol de guanábana, que constituye el mito fundacional de la organización, y aporta a establecer sentidos y significaciones de seguridad a nuevas imágenes de la mujer en la familia, la pareja y lo laboral. "Tengo que dar lo que tengo que dar ahora que lo puedo dar: ile agradezco a la Hermana! No que nos abrió los ojos, sino que nos mostró otra realidad (...), yo por lo menos tengo la oportunidad" (Consejera de Familia 1, 2009).

El árbol de guanábana es significado como un espacio propio, de escucha para las mujeres, un espacio informal para dar lugar a la palabra de sí y estar solas, su subjetividad se constituía a partir de la interacción con otras, y las normas para aprender a escucharse, protegerse entre sí a la sombra del árbol -un recurso comunitario institucionalizado cultural y sociopolíicamente en ellas y la comunidad: "Empezamos a hacer la reunión (...), antes en el árbol de guanábana, iera grandísimo! entonces nos organizamos" (Consejera de Familia 1, 2009).

Las mujeres están en proceso de empoderarse (PERKINS; ZIMMERMAN, 1995), generan habituación al decidir consolidar el espacio de escucha para usar la palabra y restituirla en lo familiar y laboral, generar justicia, exteriorizar pensamientos y sentimientos sobre su rol en la pareja o el trabajo para protegerse entre sí desde lo solidario - cooperativo y continuar el proceso de conscientización sociopolítica como sujetas de derecho. Sus formas de comunicación son metáforas, refranes, y sus experiencias, por lo tanto, el proceso de recobrar la palabra y usarla se posibilita al cuestionar las representaciones del rol de la mujer-mamá, empleada o esposa, y confrontar la actitud de su pareja.

Las normas compartidas aportan a reconstruir los lazos de protección en torno a la no violencia contra la mujer, y sus acciones son el resultado de gobernarse con nuevas simbolizaciones, interpretar la realidad y de decidir sobre los recursos comunitarios para generar bienestar. Esto es la base de la intervención para reconocer el poder de sí, por la consolidación de valores de eliminación de fuentes de opresión del género.

Período 2. ONGD 1. Consolidación-empoderamiento. Del año 1991 al año 2000. Se presenta un cambio en el rol de la líder con relación a la dirección de las acciones y hay mayor relación con agentes internacionales, donantes e instituciones, siempre conservando su autonomía en la gerencia y la intervención social. Se proyectan como un grupo de la comunidad que trabaja por el bienestar de las familias, a partir de servicios a la mujer y sistemas de protección; empiezan a ser reconocidas como una ONGD que decide colectivamente según las necesidades comunitarias, no según las visiones de donantes.

Las intervenciones sociales cambian por la emergencia de otros problemas públicos, fortaleciendo la alteridad con una estrategia cara a cara; mientras que en lo socio-organizativo aumentan las normas para asegurar protección a las mujeres y eliminar la opresión de género. Las acciones públicas son resultado del reconocimiento como sujetas políticas, su independencia de pensamiento y su economía, y se asocian a las particularidades poblacionales, se asume una posición crítica basada en la toma de la palabra y la autonomía organizativa. Las mujeres manifiestan sus sentimientos para dirigir la ONGD; se vinculan profesionales al voluntariado, y la comunidad se interesa en formarse.

En perspectiva del empoderamiento, se fortalece la conciencia crítica al cuestionar grupalmente las estrategias de intervención social usadas; se fortalece la evaluación de sus acciones; se comparten los valores de solidaridad y autonomía financiera; y se asignan roles administrativos.

Período 3. ONGD 1. Empoderamiento. Del año 2001 al año 2016. Confluyen los resultados de empoderamiento psicológico y organizacional. El primero evidente en la toma de 
decisiones, la capacidad de generar relaciones con otros y fomentar la creación de servicios de bienestar comunitario. El segundo, representado en la acción basada en la confianza con ellas, las organizaciones y la comunidad; su capacidad de ayuda basada en la escucha; usar estrategias con prácticas socioculturales comunitarias; constituir sistemas de protección en economía familiar para la autonomía financiera y la acción; y asumirse como una "organización de mujeres feministas".

Las formas de gobierno y definición de roles entorno a los problemas sociales y públicos de seguridad alimentaria y acceso a vivienda se deciden grupalmente. La autonomía organizacional, como forma de gobierno propio y de relación con la comunidad genera discrepancias y rupturas con otras ONGD's que tienen intervenciones sociales, en su opinión, asistenciales: "Trabajar en el distrito de Aguablanca es muy difícil porque la gente está esperando que le van a dar" (Consejera de Familia 4, 2016); simultáneamente, facilita la toma de decisión en la gestión de acciones. periodos.

Por su parte, en la historia biográfica organizacional de la la ONGD 2 se identifican dos

Período 1. ONGD 2. Empoderamiento. Del año 1996 al año 1999. Si bien la constitución se establece formalmente en 1996, sus raíces están en el año 1991: "nosotros hacíamos toda la militancia, en el 91 estuvimos en la constituyente, también trabajamos para que todas las cosas de las mujeres estuvieran en la constituyente" (Directiva, 2017). El proceso organizativo formal se debe a las trayectorias profesionales y personales.

en el 96 hicimos la agenda mujer. Este colectivo se conformó con amigas, muchas veníamos de militancias desde el feminismo, veníamos trabajando con mujeres. M.C. tenía un grupo que se llamaba salud mujeres, que prestaba atención médica y de orientación a mujeres. Yo había trabajado en Si Mujer. A., venía de la televisión y de medios de comunicación. Entonces nos reuníamos con mucha periodicidad, desde el 95. Ya en el 99 dijimos por qué no nos conformamos como asociación, colectivo, fundación y pensamos que efectivamente podríamos tener una fundación que nos permitiera ejecutar proyectos, teníamos una posibilidad entonces nos conformamos así en el 99 (...) todo era el activismo que hacíamos desde el feminismo, con grupos de mujeres. (Directiva, 2017)

Su configuración se posibilita por ser "un colectivo de mujeres feministas (...) entonces digamos que teníamos esas militancias personales que nos aglutinaba, que nos movía" (Directiva, 2017).

Período 2. ONGD 2. Consolidación por acción. Del año 2000 al año 2017. La consolidación de las acciones se da por el aporte comunitario de los recursos personales de las integrantes, y sus acciones son de acompañamiento en la organización de mujeres en otros lugares. Sus capacidades instaladas de formación les permitió iniciar con ese tipo de procesos de organización de base.

Allí en el 2000, en enero o febrero, alquilamos esta casa, y aquí casa una trajo su escritorio, cada una trajo un asiento de su casa, cada una trajo lo que tuvo para poder amoblar la casa, y empezamos ya a ejecutar proyectos (...) entonces ya nos fuimos conformando como colectivo. Trabajamos mucho en salud, los primeros proyectos que hicimos con la gobernación fue de demanda inclusiva en salud. (Directiva, 2017)

Las primeras intervenciones sociales se desarrollaron en torno a leer de diversas maneras lo que institucionalmente se establece en los roles de la mujer.

Se llamaba lactancia y destete, dos momentos del amor. La lactancia materna es una apuesta del estado, pero nosotras le dimos importancia al destete (...) porque no se habla de qué es una posibilidad, importante en el desarrollo de la relación madre e hijo para que haya un desarrollo armónico. Entonces hicimos una pesquisa, pensado en darle la vuelta, otras lecturas a las situaciones de las mujeres. También la violencia contra las mujeres es un tema que siempre nos ha motivado, cuando conocemos las cifras de violencia que hay, y toda la situación de las mujeres, siempre estamos pensando que se debe de hacer. (Directiva, 2017)

A diferencia de la ONGD 1, la cual presenta un proceso de empoderamiento psicológico para lograr uno organizacional y comunitario, la ONGD 2 genera capacidades de empoderamiento psicológico que potencian el comunitario.

yo creo que empoderamiento que quisiéramos, de las mujeres, es ganar autonomía yo creo que ahí la palabra autonomía es lo que yo diría que es el cimiente o lo que uno espera, que las mujeres ganemos autonomía desde muchos lugares, por supuesto la autonomía económica, una mujer puede tener autonomía económica y tener mucha dependencia afectiva, todavía mucho por hacer con respecto a lo afectivo, que podamos agenciar nuestros deseos. (Directiva, 2017)

El empoderamiento se centra en facilitar herramientas y reconocer las fortalezas de quienes participan en sus intervenciones socio participativas. Así, el empoderamiento organizacional es un medio de transformación social, como se muestra en el Cuadro 3. 
Cuadro 3

\begin{tabular}{|c|c|c|c|c|}
\hline \multicolumn{3}{|c|}{ DIMENSIÓN } & ONGD 1 & ONGD 2 \\
\hline \multirow{6}{*}{ Individual } & \multirow{3}{*}{ PROCESO } & $\begin{array}{l}\text { Aprendizaje de habilidades para la } \\
\text { toma de decisiones }\end{array}$ & $\mathrm{X}$ & \\
\hline & & Manejo de Recursos & $\mathrm{X}$ & $\mathrm{X}$ \\
\hline & & Trabajar con los demás & $\mathrm{X}$ & $\mathrm{X}$ \\
\hline & \multirow{3}{*}{ RESULTADOS } & Sentido de control & $\mathrm{X}$ & \\
\hline & & Conciencia crítica & $\mathrm{X}$ & $\mathrm{X}$ \\
\hline & & Comportamiento participativo & $\mathrm{X}$ & $\mathrm{X}$ \\
\hline \multirow{6}{*}{ Organizacional } & \multirow{3}{*}{ PROCESO } & $\begin{array}{l}\text { Oportunidades en participar en la } \\
\text { toma de decisiones }\end{array}$ & $\mathrm{X}$ & $\mathrm{X}$ \\
\hline & & Responsabilidades compartidas & $\mathrm{X}$ & $\mathrm{X}$ \\
\hline & & Liderazgo compartido & $\mathrm{X}$ & $\mathrm{X}$ \\
\hline & \multirow{3}{*}{ RESULTADOS } & $\begin{array}{l}\text { Competencia efectiva en manejo } \\
\text { de recursos }\end{array}$ & $\mathrm{X}$ & \\
\hline & & $\begin{array}{l}\text { Redes de trabajo: coaliciones } \\
\text { entre organizaciones }\end{array}$ & $\mathrm{X}$ & $\mathrm{X}$ \\
\hline & & Influencia política & $\mathrm{X}$ & $\mathrm{X}$ \\
\hline \multirow{6}{*}{ Comunitario } & \multirow{3}{*}{ PROCESO } & $\begin{array}{l}\text { Acceso a recursos de la } \\
\text { comunidad }\end{array}$ & $\mathrm{X}$ & \\
\hline & & $\begin{array}{l}\text { Apertura de estructuras } \\
\text { mediadoras }\end{array}$ & $\mathrm{X}$ & $\mathrm{X}$ \\
\hline & & Tolerancia a la diversidad & $\mathrm{X}$ & $\mathrm{X}$ \\
\hline & \multirow{3}{*}{ RESULTADOS } & Coaliciones organizacionales & $\mathrm{X}$ & $\mathrm{X}$ \\
\hline & & Liderazgo plural & $\mathrm{X}$ & $\mathrm{X}$ \\
\hline & & $\begin{array}{l}\text { Habilidades participativas de las } \\
\text { residentes comunitarias }\end{array}$ & $\mathrm{X}$ & \\
\hline
\end{tabular}

Fuente: Elaboración propia.

\#PraCegoVer Cuadro 3 presenta tres columnas, Dimensión/Proceso y Resultado de Potenciación, ONGD1 y ONGD2, con las conductas de cada una de las ONGDs, en nivel subjetivo. Columna 1 se subdivide en tres, cada una a la vez subdividida en Proceso y Resultados, con sus propias subdivisiones para cada proceso y resultados y marcación en X para cada ONGD (si cumple o no con el requisito)

\section{Organizaciones feministas libres de opresión de género}

Las organizaciones estudiadas no conservan una forma de burocracia feminista (ASHCRAFT, 2001) ni son genderizadas. Las formas de organizarse y de actuar sobre los problemas sociales son agenciales y de colaboración intraorganizacional (O'OOLE; MONTJOY, 1984), la distribución y uso de recursos se da por estructuras igualitarias, se gobiernan a sí mismas y con autoridad descentralizada, no limitan su actuar por procesos burocráticos y políticos (DELEON; DELEON, 2002), son estratégicas en función de sus principios feministas (Teología de la liberación de la opresión de género o el feminismo), incorporan elementos organizacionales irónicos ante las demandas de la lógica administrativa de las ONGD's con relación a los donantes, deciden por consensos colectivos (RIGER, 1994) y emotivos, y critican las intervenciones sociales, su alcance y enfoque. Sus intervenciones sociales comienzan con la satisfacción o no de necesidades y la calidad de vida comunitaria, para: a) construir la identidad propia a partir de la identificación de factores influyente en el problema o conflicto; b) crear espacios para fortalecer la toma de decisión y autonomía; c) desarrollar habilidades socio-cognitivas en el ámbito afectivo-relacional del individuo para descubrir e investigar los intereses y posibilidades personales; y plantear con las mujeres de base los nuevos retos o metas, según la reflexividad sobre su agencia. 
La implementación se realiza por construcciones relacionales basadas en prácticas deliberativas (FOLLETT, 1918), las cuales posibilitan identificar los problemas públicos y sociales con dispositivos construidos a partir de sus recursos culturales, sociales y psicológicos (lo hacen en terreno caminando, conversando, escuchando); movilizar la comunidad y sostener las redes de protección de las acciones desarrolladas; organizarse en torno a los problemas públicos; y liberar y restituir el rol y la palabra de la mujer comunitariamente. En los barrios logran cambios en el bienestar por procesos de concientización sociopolítica de las mujeres y de sí como sujeto psicológico en el tiempo; resolviendo los problemas de manera eficiente, pragmática y única, dado que cada barrio/comunidad presenta procesos colectivos únicos y con su propia forma de gobernarse (ELIAS, 2010).

Las mujeres han enfrentado dilemas organizacionales por la comprensión que hacen de los problemas públicos en las políticas top down, así como, del proceso de identificación, recreación de ideas y síntesis de la interacción humana para empoderar (ELIAS, 2010) a la comunidad, demarcando su rol como sujetas históricas. Pero han generado tácticas basadas en espacios de sensibilidad emocional para expresar actitudes producto de la demanda afectiva, de la consciencia sobre su discurso feminista y la acción; para resolver las tensiones con los donantes y la comunidad. En reuniones y eventos adoptan diversas formas comunicativas que expresan sus frustraciones, alegrías, iras, tristezas (MUMBY; PUTNAM, 1992) y, en las relaciones, critican tanto el espacio habitado y de ejecución de las acciones públicas (FORESTER, 2008) como la transferibilidad de políticas. La alegría como emoción y la felicidad como sentimiento acompañan la ejecución de las acciones, constituyendo un aprendizaje cotidiano significativo basado en la acción comunitaria. La acción de las mujeres cuestiona la desigualdad, obteniendo una consciencia histórica de su rol en el desarrollo.

Tal posición política ha tenido un costo práctico (ASHCRAFT, 2001), pero no ético, pues incorporan las reconfiguraciones de las necesidades individuales en las prácticas formales y las emociones, pero no reprimen a sus integrantes, desarrollan habilidades y cooperan (RIGER, 1994) para afrontar los dilemas por conflictos entre necesidades individuales y organizacionales, sin ceder sus ideologías. Es decir, las emociones son actos relacionales entre mujeres para asegurar sus acciones públicas, se configura un campo de no planificación, espontaneidad, autenticidad, contextualización cultural que garantiza la vida social de la organización, y dota de significado las acciones conjuntas. Esto supera el carácter coordinador de las acciones, casi siempre asignadas a las organizaciones feministas solo por sus valores.

El empoderamiento es central en las intervenciones sociales, en la ONGD 1 lo denominan fortalecimiento (MONTERO, 2009) y tiene como base una relación bidireccional entre lo individual y lo comunitario; mientras que la ONGD 2 potencia el comunitario, por ser referente e instalar capacidades. Sus procesos transformadores se basan en la acción comunitaria e impactan el desarrollo social (PERKINS; ZIMMERMAN, 1995), pues se adaptan a los contextos y los problemas públicos/sociales comunitarios, usando formas de acción colaborativas, no competitivas, por lo cual no son genderizadas. Trascienden ese tipo pues generan conscientización política, emancipación en las mayorías populares y cambio social, no reproducen las diferencias de género opresoras (BRITTON, 2000) y son particulares en las 'formas organizacionales feministas' que asumen en la implementación de políticas. Rol con el cual se superponen a la relación individuo-sociedad en un estado de mercado, y se oponen a la ongenización, dándole relevancia a la colaboración y la dimensión subjetiva política de las mujeres basada en empoderamiento psicológico y comunitario.

Las formas de organización de las mujeres para su accionar público, denominadas en la investigación como formas organizacionales feministas, son esquemas narrativos desde los cuales se constituye un evento de la realidad como un fenómeno perceptible y con sentido, desde cierta perspectiva, es decir, en la promoción de una estética de la realidad, una manera de vivir, de relacionarse con los otros, de significar la implementación de políticas públicas y el bienestar. Evidencian la posición subjetiva ante las demandas externas que cuestionan sus ideologías feministas y la visión del rol como implementadoras; así como su posición política ante el carácter simbólico de las políticas en diferentes problemas y los procesos de significación (FORESTER, 2008). Tales formas son matrices narrativas complejas configuradas y divulgadas a través de los diferentes mecanismos y dinámicas de la vida organizacional, es decir, del sentido de la vida organizacional (OSWICK; PUTNAM; KEENOY, 2004), hacen parte de la historicidad de las mujeres, del origen de la organización y se organizan, modifican, dinamizan y actualizan de acuerdo con ciertos escenarios de acción pública de las mujeres. Son un efecto histórico del entramado simbólico de la implementación de políticas en el sujeto en cuatro subtipos de la forma organizacional feminista, estructurados según la concepción de la implementación, origen y visión del problema público, y la acción de las mujeres.

Para la forma organizacional feminista, la implementación es una disfunción estructural complejizada por factores secundarios psicológicos y contextuales, en la cual el problema comunitario no concuerda con el de la implementación, pero es susceptible de ser intervenido por el de control 
que tienen las mujeres sobre las necesidades sociales, los recursos, y los cursos de acción; en los subtipos: orgánico-público, tiene acciones validados y basados en emociones y sentimientos de alegría o felicidad al ejecutar la política pública, implica asumir las consecuencias de rechazar o terminar la ejecución de políticas; orgánico popular-comunitaria, en el que las formas de organización son validados por su saber, producto de experiencias personal y colectiva; emocional, se toman decisiones según sentimientos y emociones generados por la implementación de política; y existencial, orientadas a diversas psicoterapias basadas en lo lúdico o artísticas y los cambios de estilos.

En suma, estas ONGD no tienen formas híbridas burocráticas. Al ser feministas, sus formas de acción se basan en relaciones con rupturas psicológicas sobre cómo son los problemas públicos y el conflicto, para lograr coordinación sincrónica o armónicas por coherencia entre sus valores feministas y un empoderamiento orientado políticamente a la liberación y la consciencia crítica; además, usan las emociones para generar compromiso, cooperación y racionalidad comunitaria de las mujeres contextualizada e históricamente circunscritas.

\section{Conclusión}

La investigación evidencia que la agenda política, los problemas sociales/públicos y el empoderamiento psicológico y organizacional de la comunidad se dan, pues las mujeres reconocen su realidad, desarrollan autonomía, comparten normas con la comunidad, y definen sus valores (p.e. la teoría de la liberación aplicada a la eliminación de la opresión de género), estructura, distribución de recursos y enfoque de la intervención. Al coexistir con organizaciones de los movimientos sociales, develan y cuestionan las diferencias estructurales y culturales con relación a la opresión política y personal, la invisibilidad de la mujer producto del lenguaje genderizado y naturalizado, y la institucionalización del empoderamiento en las políticas. En ambas ONGD's, los procesos y productos de empoderamiento psicológico son transversales al organizacional y comunitario. Son las mujeres en sus formas de coordinación sobre los problemas sociales quienes resuelven los conflictos sociales y hacia su interior, generando formas feministas de organización singulares

Por último, los estudios de implementación de políticas públicas asociados a los estudios de las organizaciones y de género permiten establecer la sostenibilidad de los procesos de intervención social participativo, en una visión de desarrollo centrado en la mujer post-empoderada con organizaciones integradas por ellas, sin oprimir sus valores feministas en un estado neoliberal, sus formas narrativas y acciones para solucionar problemas.

\section{Referencias}

ACKER, Joan. "Hierarchies, jobs, bodies: A theory of gendered organizations". Gender and Society, v. 4, n. 2, p. 139-158, 1990. Disponible en http://www3.kau.se/kurstorg/files/a/C10B963709d16168 ABYyY14FAA27/Ack_Hierarchies.pdf. Acceso el 08/08/2014.

ACKER, Joan. "Gender and organizations". In: SALTZMAN CHAFETZ, Janet (Ed.), Hanbook of the sociology of gender, New York: Springer, 2006. p. 177-194.

ALVAREZ, Sonia. "Advocating feminism: The Latin American Feminist NGO "Boom"'. International Feminist Journal of Politics, v. 1, n. 2, p. 181-209, 1999. Disponible en https://doi.org/10.1080/14616749935 9880. Acceso el 26/09/2015.

ALVESSON, Matts; STANLEY, David. "Critical Theory and Postmodernism Approaches to Organizational Studies”. In: CLEGG, Stewart R.; HARDY, Cynthia; LAWRENCE, Thomas B.; NORD, Walter R.. (Eds.), The SAGE Handbook of Organization Studies (Second edition ed.). New York: SAGE, 2006. p. 255-283.

ASHCRAFT, Karen Lee. Gender and Diversity: Other Ways to "Make a Difference". In: ALVESSON, Matt; BRIDGMAN; Willmott, H (Eds.), The Oxford Handbook of Critical Management Studies. United States: Oxford University Press, 2009.

ASHCRAFT, Karen Lee. "Organized Dissonance: Feminist Bureaucracy as Hybrid Form”. The Academy of Management Journal, v. 44, n. 6, p. 1301-1322, 2001.

BAMBERGER, Michael; PODEMS, Donna. "Feminist evaluation in the International Development context”. New Directions for evaluation, v. 96, p. 83-96, 2002.

BARTUNEK, Jean; WALSH, Kate; LACEY, Catherine. "Dynamics and Dilemmas of Women Leading Women". Organization Science, v. 11, n. 6, p. 589-610, 2000.

BLACK, Cyril; BURKE, John. "Organizational partipation and poblic policy". World Politics, v. 35, n. 3 , p. 393-425, 1983. Disponible en https://doi.org/10.2307/2010159. Acceso el 13/06/2014. 
BRITTON, Danna. "The epistemology of the gendered organizations". Gender and Society, v. 14, n. 3 , p. 418-434, 2000. Disponible en https://doi.org/10.1177/089124300014003004. Acceso el 13/10/ 2014.

COSGROVE, Lisa; MCHUGH, Maureen. "Speaking for ourselves: Feminist methods and community psychology". American Journal of Community Psychology, v. 28, n. 6, p. 815-837, 2000.

DELEON, Peter; DELEON, Linda. "What ever happened to implementation? An Alternative Approach". Journal of Public Administration Research and Theory: J-PART, v. 12, n. 4, p. 467-492, 2002.

EDWARDS, Michael; HULME, David. "Too close for comfort? The impact of official aid on nongovernmental organizations". World Development, v. 6, n. 24, p. 961-973, 1996.

ELIAS, María. "Governance from te ground up: Rediscovering Mary Parker Follett". Public Administration and Management, v. 15, n. 1, p. 19-45, 2010.

FARAH, Martha. "Genero e poliiticas puiblicas". Revista Estudos Feministas, v. 12, n. 1, p. 47-71, 2004.

FAWCETT, Stephen; PAINE-ANDREWS, Adrienne. "Working Together for Healthier Communities: A Research-Based Memorandum of Collaboration". Public Health Reports, Supplement on Healthy Cities/Healthy Communities, 1999.

FOLLETT, Mary Parker. "Community is a process". The Philosophical Review, v. 28, n. 6, p. 576-588, 1919. Disponible en https://www.jstor.org/stable/21 78307. Acceso el 06/03/2015.

FOLLETT, Mary Parker. The New State: Group Organization, the Solution of Popular Government, 1918. Disponible en http://sunsite.utk.edu/FINS/Mary_Parker_Follett/Fins-MPF-01.html. Acceso el 24/ $03 / 2015$.

FOLLETT, Mary Parker. "Constructive conflict". In: METCALF, Henry C.; URWICK, L. (Eds.). Dynamic administration: The collected papers of Mary Parker Follett. (Second published 1942) New York, NY: Harper \& Brothers Publishers, 1925. p. 30-49.

FORESTER, John. "Policy Anlysis as critical listening". In: MORAN, Michael; MARTIN, Rein; GOODIN; Robert E. (Ed.). The Oxford Handbook of Public policy (First published in paperback ed., p. 124-151). New York, United States: Oxford University Press, 2008.

FOWLER, Alan. "NGO futures. beyond aid: NGDO values and the fourth position". Third World Quaterly, v. 21 , n. 4 , p. $589-603,2000$.

HILL, Michael; HUPE, Peter. "The future of implementation studies". In: HILL, Michael; HUPE, Peter (Eds.). Implementing public policy (First ed., p. 112), SAGE Publications, 2002.

LANGLEY, Ann. "Strategies for Theorizing from process data". The Academy of Management Review, v. 24, n. 4, p. 691-710, 1999.

MARTIN, Joanne. "Deconstructing organizational taboos: The suppression of gender conflict in organizations". Organization Science, v. 1, p. 339-359, 1990.

MARTIN, Patricia Yancey. "Rethinking Feminist Organizations". Gender and Society, v. 4, n. 2, p. 182206, 1990.

MEJÍA, Juliana. "Modelos de implementación de las políticas públicas en Colombia y su impacto en el bienestar social". Analecta política, v. 2, n. 3, p. 141-164, 2012.

MISOCZKY, Maria Ceci Araújo; FLORES, Rafael Kruter; SILVA, Sueli Maria Goulart. "Estudos organizacionais e movimentos sociais: o que sabemos? Para onde vamos?" Cadernos EBAPE.BR, Fundação Getúlio Vargas, v. 6, n. 3, p. 1-14, 2008.

MONTERO, Maritza. "Why a special section about community psychology and politics". American Journal of Community Psychology, v. 43, n. 1-2, p. 98, 2009.

MUMBY, Dennis; PUTNAM, Linda. "The politics of emotion: A feminist teading of bounded rationality". The Academy of Management Journal, v. 17, n. 3, p. 465-486, 1992.

NATIVIDAD, Elisângela Abreu; PEREIRA, José Roberto; OLIVEIRA, Vânia Aparecida Rezende de. "Gestão de política pública de geração de trabalho e renda: uma análise do programa nacional de 
incubadoras de cooperativas populares". Revista de Políticas Públicas, São Luis, v. 17, n. 1, p. 241 250, 2013. Disponible en http://www.periodicoseletronicos.ufma.br/index.php/rppublica/article/view/ 1744/571. Acceso el 24/11/2014.

NEIDELL, Shara. "Women's empowerment as a public problem: A case study of the 1994 International Conference on Population an Development". Population Research and Policy Review, p. 247-260, 1998.

OLIVEIRA, Lucas Canestri de; PEREIRA, José Roberto; FERREIRA, Patricia Aparecida. "Análise estruturacionista de políticas públicas: a dualidade da política pública". Revista de Políticas Públicas, São Luis, v. 17, n. 1, p. 27-36, 2013.

OSWICK, Cliff; PUTNAM, Linda; KEENOY, Tom. Tropes, discourse and organizing. In: GRANT, David; HARDY, Cynthia; OSWICK, Cliff; PUTMAN, Linda (Eds.), The SAGE Handbook of Organizational discourse, London: SAGE Publications, 2004, p. 105-128.

O'TOOLE, Laurence; MONTJOY, Robert. "Interorganizational policy implementation: A theoretical perspective". Public Administration Review, v. 44, n. 6, p. 491-503, 1984.

PEARCE, Jenny. "Power and the Twenty first Century Activist: From the Neighbourhood to the Square". Development and Change, v. 44, n. 3, p. 639-663, 2013.

PERKINS, David; ZIMMERMAN, Mark. "Empowerment theory, research, and application". American Journal of Community Psychology, v. 23, n. 5, p. 569-579, 1995. Disponible en https://my.vanderbilt. edu/perkins/files/201 1/09/empintro.proquest.pdf. Acceso el 10 de abril de 2015.

RIGER, Stephanie. "Challenges of Success: Stages of Growt in feminist Organizations". Feminist Studies, v, 20, n. 2, p. 275-300, 1994.

SARDENBERG, Cecília. "Liberal vs. Liberating empowerment: a latinamerican feminist perspective on conceptualising women's empowermet”. IDS Bulletin, v. 39, n. 6, p. 18-27, 2008. Disponible en https://core.ac.uk/download/pdf/43539239.pdf. Acceso el 18/06/2014.

SILVA, Carlos Rodrigues da. "Açöes afirmativas: uma proposta de superação de racismo e das desigualdades". Revista de Políticas Públicas, São Luis, v. 14, n. 1, p. 67-76, 2010. Disponible en http://www.periodicoseletronicos.ufma.br/index.php/rppublica/article/viewFile/354/773. Acceso el $18 / 01 / 2014$.

YIN, Robert. Case study research: design and methods (Tercera ed. Vol. 5). USA: Sage publications, 2003.

Silvia Cristina Caicedo-Muñoz (sccaiced@usbcali.edu.co) es Doctora en Administración, en la línea de Gobierno y Políticas Públicas, y Magister em Políticas Públicas de la Universidad del Valle, Colombia. Investigadora Junior Colciencias, Colombia. Docente de pregrado en Psicología de la Universidad de San Buenaventura, Cali y catedrática invitada en la Maestría en Políticas Públicas de la Universidad del Valle, Colombia.

\section{COMO CITAR ESTE ARTÍCULO, DE ACUERDO CON LAS NORMAS DE LA REVISTA:}

CAICEDO-MUÑOZ, Silvia Cristina. "Formas organizacionales feministas en la implementación de políticas públicas". Revista Estudos Feministas, Florianópolis, v. 27, n. 3, e5721 1, 2019.

\section{CONTRIBUCIÓN DE AUTORÍA}

Silvia Cristina Caicedo-Muñoz: El artículo es producto de la tesis doctoral en Administración, en la línea de Gobierno y Políticas Públicas, por ende, la participación de autoría en el artículo es en la estructuración, recolección de información y su respectivo análisis, así como en la elaboración, redacción y discusión de resultados.

\section{FINANCIACIÓN}

No aplicable. 
CONSENTIMIENTO DE USO DE IMAGEM

No aplicable.

APROBACIÓN DE COMITÉ DE ÉTICA EN INVESTIGACIÓN

No aplicable.

\section{CONFLICTO DE INTERESES}

No aplicable.

\section{LICENCIA DE USO}

Este artículo está licenciado bajo la Licencia Creative Commons CC-BY Internacional. Con esta licencia se puede compartir, adaptar, crear material para cualquier objetivo, siempre que se le atribuya la autoría.

HISTORIAL

Recebido em 23/05/2018

Reapresentado em 12/1 1/2018

Aprovado em 30/04/2019 